\title{
Fatores preditores de resultados no tratamento do transtorno obsessivo-compulsivo com as terapias comportamental e cognitivo-comportamental: uma revisão sistemática*
}

\section{Outcome predictor factors in the treatment of obsessive-compulsive disorder using behavior and cognitive-behavior therapies: a systematic review}

\section{Andréa Litvin Raffin'; Ygor Arzeno Ferrão' '; Fernanda Pasquoto de Souza'"I; Aristides Volpato Cordioli'}

'Psicóloga. Aluna, Curso de Pós-Graduação: Ciências Médicas, Departamento de Psiquiatria, Universidade Federal do Rio Grande do Sul (UFRGS), Porto Alegre, RS.

"Médico psiquiatra. MSc, PhD. Professor, Programa de Pós-Graduação, Centro Universitário Metodista Instituto Porto Alegre, RS.

I'Psicóloga. Aluna, Curso de Pós-Graduação: Ciências Médicas, Departamento de Psiquiatria, UFRGS.

IVMédico psiquiatra. MSc, PhD. Professor ajunto, Departamento de Psiquiatria e Medicina Legal, UFRGS. Coordenador, Programa de Transtornos de Ansiedade (PROTAN), Hospital de Clínicas de Porto Alegre (HCPA), Porto Alegre, RS.

\section{Endereço para correspondência}

Rev Psiquiatr RS. 2008; 30(1 Supl).

\section{RESUMO}

I NTRODUÇÃO: As terapias comportamental e cognitivo-comportamental reduzem os sintomas do transtorno obsessivo-compulsivo em mais de $70 \%$ dos pacientes. Entretanto, cerca de $30 \%$ não obtêm nenhuma melhora. Conhecer fatores associados a esses desfechos poderia auxiliar numa melhor indicação do tratamento, incrementando sua eficácia.

MÉTODO: Foram revisados trabalhos que investigaram fatores preditivos de resultados nos 
tratamentos do transtorno obsessivo-compulsivo, nas fontes PubMed, Psycl NFO e LI LACS. Termos utilizados na busca: predictive factors OR prediction AND obsessive-compulsive disorder AND exposure response prevention OR ritual prevention OR behav* therapy OR cognitive behav* therapy, resultando em 104 artigos. As referências dos artigos foram analisadas, possibilitando maior número de trabalhos revisados. Excluíram-se artigos que utilizaram apenas farmacoterapia e aqueles que não abordavam o tema, resultando em 29 artigos.

DI SCUSSÃO: As variáveis demográficas parecem exercer papel indireto, destacando-se sexo masculino e ausência de companheiro(a) como fatores de pior prognóstico. Maior gravidade e início precoce dos sintomas indicariam piores resultados. O transtorno esquizotípico comórbido é potencialmente negativo. Sintomas relacionados ao colecionismo e com conteúdo sexual/religioso indicam pior prognóstico. Maior nível de insight, motivação e colaboração com o tratamento são características favoráveis. Maior intensidade da melhora e a remissão completa são preditores para a não-recaída.

CONCLUSÃO: A identificação de fatores preditores de resultados está longe de ser esclarecida. É possível que múltiplos fatores, cujas associações são complexas, atuem em conjunto. Os resultados podem depender de fatores não-específicos, ainda não abordados pelos estudos. A heterogeneidade do transtorno obsessivo-compulsivo e das pesquisas e o uso associado de medicamentos dificultam o estabelecimento de conclusões mais definidas.

Descritores: Prognóstico, transtorno obsessivo-compulsivo, terapia cognitivo-comportamental, terapia comportamental, exposição e prevenção de rituais.

\section{ABSTRACT}

I NTRODUCTI ON: Behavioral and cognitive-behavioral therapies reduce symptoms of obsessivecompulsive disorder in over $70 \%$ of patients. However, about $30 \%$ do not show any improvement. The knowledge of factors associated with these outcomes may inform better treatment indications and improve treatment efficacy.

METHOD: Review of studies that investigated predictors of obsessive-compulsive disorder treatment results in PubMed, PsychoINFO and LI LACS databases. Terms used in the search were predictive factors OR prediction AND obsessive-compulsive disorder AND exposure response prevention OR ritual prevention OR behav* therapy OR cognitive behav* therapy." The search retrieved 104 studies. The references of retrieved studies were also analyzed to ensure that all relevant studies were included. Studies that used only pharmacotherapy or did not discuss the topic under analysis were excluded, and 29 studies met inclusion criteria.

DI SCUSSI ON: Demographic variables seem to play an indirect role in treatment results; male sex and not having partner are factors associated with poor prognosis. Greater severity and early symptom onset also indicate poor outcomes. Comorbid schizotypal disorder is potentially negative. Symptoms associated with hoarding and sexual/religious obsessions predict poor prognosis. Greater insight, motivation and collaboration with treatment are favorable characteristics. Greater improvement and complete remission of symptoms are predictors of no relapse.

CONCLUSION: The identification of outcome predictors is still far from complete. Multiple factors may contribute to results, and their associations are complex. Results may depend on unspecific factors not yet investigated. Definite conclusions are difficult to reach because of the heterogeneity of obsessive-compulsive disorder and of the studies investigating it and the combined use of drugs.

Keywords: Prognosis, obsessive-compulsive disorder, cognitive-behavior therapy, behavior therapy, exposure and response prevention. 


\section{NTRODUÇÃO}

O transtorno obsessivo-compulsivo (TOC) possui curso crônico e é caracterizado pela presença de obsessões e compulsões, que ocupam boa parte do tempo do paciente, acompanhadas de desconforto, comprometendo as rotinas diárias, o desempenho profissional e as relações interpessoais ${ }^{1}$.

Considerado raro até bem pouco tempo, verificou-se que o TOC é bastante comum, com uma prevalência entre $1,6 \%$ e $3,1 \%$ da população em algum momento da vida ${ }^{2}$. Acomete em geral pessoas jovens, ao final da adolescência. É um transtorno grave, que incapacita cerca de $10 \%$ dos seus portadores ${ }^{3}$.

São considerados tratamentos de primeira linha a clomipramina, os inibidores seletivos da recaptação da serotonina (ISRS), a terapia comportamental (TC) de exposição e prevenção de rituais (EPR) e a terapia cognitivo-comportamental (TCC) ${ }^{4}$.

Os medicamentos são a terapia de escolha em pacientes com pouco juízo crítico (insight) sobre a doença ou nas situações em que os sintomas do TOC são muito graves. Entretanto, a redução na intensidade dos sintomas obsessivo-compulsivos (SOC) é parcial, entre 30 e $40 \%$ em média, e apenas $20 \%$ dos pacientes conseguem uma remissão completa ${ }^{5-7}$. As recaídas são freqüentes, ocorrendo em cerca de $80 \%$ dos pacientes depois de 2 meses de interrupção dos medicamentos ${ }^{8}$. A intolerância aos efeitos adversos, principalmente com o uso da clomipramina, bem como a nãoaceitação ou o abandono do tratamento, pode chegar a $20 \%^{7}$.

Desde os anos 70, a terapia de EPR foi introduzida no tratamento do TOC, sendo eficaz em mais de $70 \%$ dos pacientes que aderem ao tratamento ${ }^{9,10}$. Constitui-se, junto com os medicamentos, um dos tratamentos de escolha para o TOC. Estudos mais recentes ${ }^{11,12}$ têm demonstrado sua superioridade em relação aos medicamentos, especialmente na intensidade da redução das compulsões e no percentual de pacientes que obtêm remissão completa dos sintomas ${ }^{12}$ - condição associada a não-recaídas no seguimento ${ }^{13}$. Além das técnicas de EPR, são utilizadas técnicas cognitivas que auxiliam na correção das crenças distorcidas e proporcionam melhor compreensão dos sintomas do TOC. Ambas podem ser vistas como complementares, sendo possível utilizá-las em conjunto sob a forma da TCC. A falta de disponibilidade das psicoterapias, bem como seu custo elevado, resultou na proposta de oferecê-las na modalidade em grupo, permitindo tratamento de um número maior de pessoas e o acréscimo de fatores grupais, melhorando o comprometimento dos pacientes nas tarefas de EPR. Os tratamentos em grupo conferem resultados similares aos individuais ${ }^{14,15}$ - Cordioli et al. ${ }^{16}$, em um ensaio clínico randomizado, concluíram que a eficácia em reduzir a intensidade dos SOC com a TCC em grupo é de cerca de $70 \%$.

Entretanto, nem todos os pacientes de TOC beneficiam-se com as psicoterapias. Um grupo apresenta uma resposta rápida, com remissão completa dos sintomas, enquanto outro, ao redor de $30 \%$, não responde ao tratamento ${ }^{16-18}$. É ainda comum a resposta parcial, em que os pacientes, embora melhorem, ainda apresentam sintomas residuais da doença. Eles têm maior risco de recaídas ao longo do período de até 2 anos após o tratamento ${ }^{13,17}$. Esclarecer essas questões permitiria compreender melhor a natureza do TOC e os fatores que agravam os sintomas e dificultam sua remoção. Permitiria, ainda, melhorar os critérios de indicação, auxiliando no desenvolvimento de novos recursos e estratégias para pacientes refratários.

O presente trabalho tem por objetivo elaborar uma revisão dos estudos de identificação de fatores preditores de resposta às TC e TCC para o TOC em adultos (a partir dos 18 anos de idade). As características dos pacientes que não se beneficiam e o fator que os distingue dos que aproveitam os tratamentos são questões ainda em aberto e objeto da presente revisão bibliográfica. 


\section{MÉTODO}

Foi realizada uma pesquisa bibliográfica sistemática, de 1980 a maio de 2007, tendo-se como fontes as bases de dados PubMed, Psycl NFO e LILACS. A pesquisa utilizou os seguintes descritores: predictive factors OR prediction AND obsessive-compulsive disorder AND exposure response prevention OR exposure ritual prevention; predictive factors OR prediction AND obsessivecompulsive disorder AND behav* therapy; predictive factors OR prediction AND obsessivecompulsive disorder AND cognitive behav* therapy. Foram listados 184 artigos, sendo 80 em duplicidade, resultando, a busca, em 104 artigos. Foram excluídos os trabalhos cuja amostra era composta por crianças e adolescentes (indivíduos menores de 18 anos); aqueles que, embora tenham sido listados pela pesquisa bibliográfica, não abordavam o tema proposto; e os que utilizaram como tratamento do TOC apenas a farmacoterapia. Foram incluídos os trabalhos cuja terapêutica envolveu abordagem medicamentosa associada às terapias de EPR e TCC, já que estes se constituem na maioria dos estudos. As referências citadas nos artigos foram objeto de análise, possibilitando maior número de trabalhos revisados. Foram selecionados 29 estudos, apresentados na Tabela 1. 
Tabela 1- Estudos sobre preditores de resposta no tratamento de pacientes com TOC

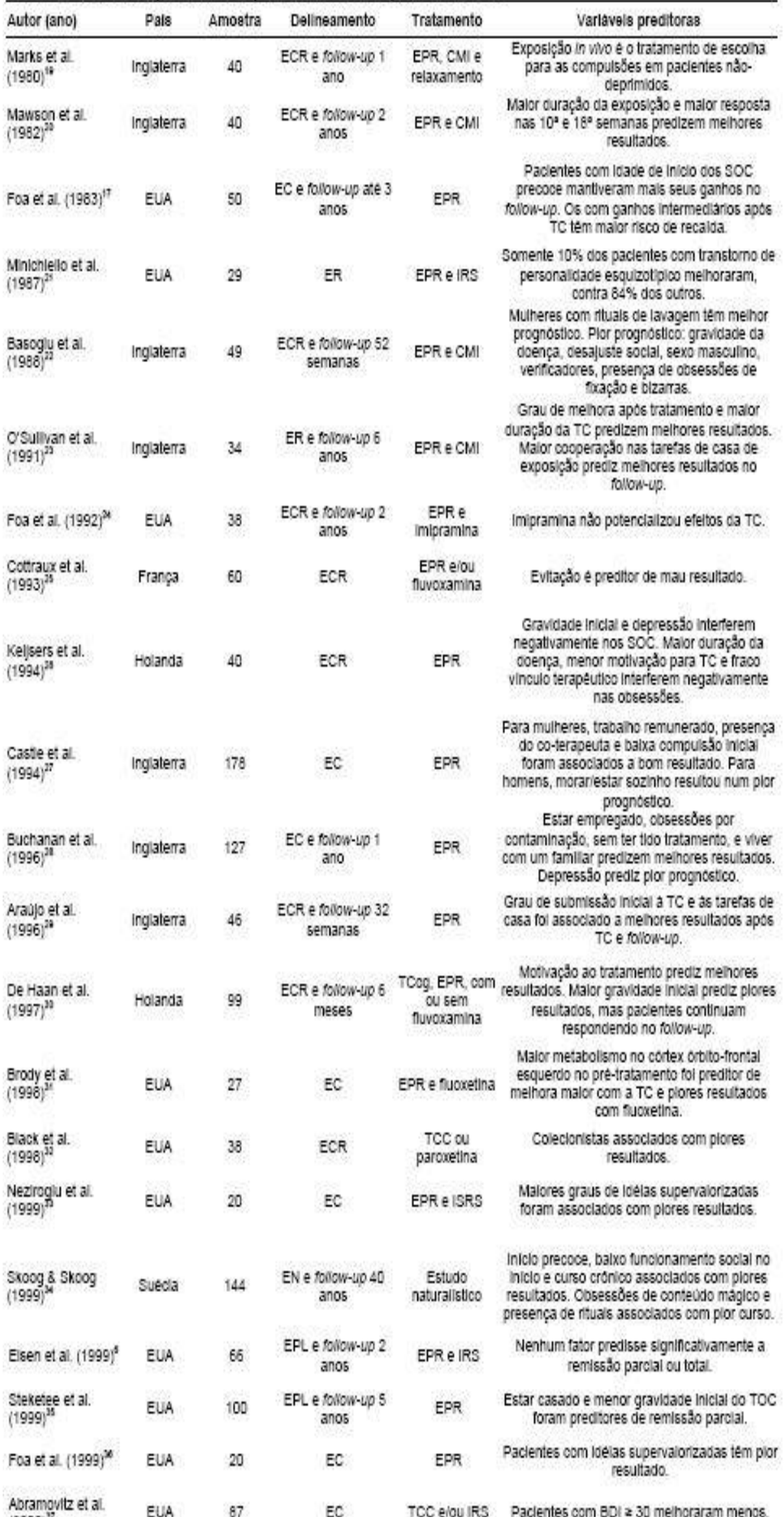




\title{
RESULTADOS/ DI SCUSSÃO
}

\author{
Fatores sociodemográficos
}

Gênero

Basoglu et al. ${ }^{22}$, através de um ensaio clínico randomizado, analisaram preditores de melhora do TOC em pacientes tratados com TC e clomipramina. Avaliaram 49 pacientes ambulatoriais, na faixa dos 18 aos 60 anos. Os pacientes foram vinculados aleatoriamente às condições de tratamento, com duração entre 17 e 23 semanas. Através de uma análise de regressão múltipla, o sexo masculino $(n=27)$, entre outras variáveis, apareceu como fator preditor de mau resultado aos tratamentos.

Castle et al. ${ }^{27}$ analisaram preditores de resultado à TC em 178 pacientes ambulatoriais, tratados entre 1977 e 1989. Encontraram diferenças entre os sexos, já que, para os homens $(n=75)$, morar sozinho ou viver só foi preditor de mau resultado. Para as mulheres $(n=103)$, possuir trabalho remunerado na época do tratamento, ter um co-terapeuta envolvido (parente, amigo ou assistente social com conhecimentos sobre os princípios da terapia, auxiliando nas tarefas de casa de exposição) e apresentar baixos escores iniciais na listagem de compulsões foram preditores associados a um bom resultado à TC.

Por outro lado, Araújo et al. ${ }^{29}$, em um ensaio clínico randomizado, não identificaram o gênero como possível preditor de resultado no tratamento de 46 pacientes ambulatoriais com TOC, tratados por 9 semanas com duas formas de terapia de EPR (exposição in vivo ou imaginária + in vivo).

McLean et al. ${ }^{39}$, em um ensaio clínico randomizado, compararam os efeitos da TCC e da TC no tratamento do TOC, ambas na modalidade em grupo. Também não observaram o gênero como uma variável preditora de resultado às terapias. Dos 76 pacientes que começaram os tratamentos, 63 o completaram ( 31 na TCC e 32 na TC), sendo 33 homens e 30 mulheres.

Estudos sugerem que o gênero pode exercer papel indireto na resposta aos tratamentos, devido, provavelmente, às diferenças biológicas entre homens e mulheres. O sexo feminino foi associado a maior gravidade dos sintomas depressivos ${ }^{45}$; já o sexo masculino apresentou menor idade de início dos SOC, maior gravidade basal, maior número de obsessões e compulsões e pior funcionamento global $^{45}$.

\section{Situação conjugal}

Foa et al. ${ }^{17}$ não verificaram associação significativa entre o resultado da terapia e o modo de vida (viver sozinho ou com outras pessoas) ou o estado civil. Foram analisados dados de 50 pacientes com TOC, os quais receberam tratamento com a técnica de EPR em 12 sessões de 2 horas.

Por outro lado, Steketee et al. ${ }^{35}$, em um estudo prospectivo, analisaram dados de 100 pacientes com TOC, de um total de 711 adultos, participantes do Harvard/Brown Anxiety Disorders Research Project, com o objetivo de identificar preditores de curso do TOC. Na amostra analisada, $43 \%$ dos pacientes eram casados ou tinham companheiro(a), $41 \%$ dos pacientes eram solitários, e $16 \%$ dos 
pacientes viviam com outras pessoas. Os pacientes casados tiveram o dobro da probabilidade de apresentarem remissão parcial dos sintomas em um período de 5 anos em comparação com os solteiros, constituindo-se a situação conjugal um forte preditor de resultado à TC.

A condição de ter companheiro(a) poderia ser em decorrência de esses indivíduos apresentarem um subtipo menos grave de TOC, com maiores chances de estabelecer relacionamentos. Contudo, não fica evidente se o TOC refratário faz os pacientes ficarem sem cônjuge (causa) ou se estar sem cônjuge é fator preditivo de má resposta ao tratamento (efeito).

Situação socioeconômica e educacional, nível de adaptação social

De Haan et al. ${ }^{30}$, em um ensaio clínico randomizado, analisaram preditores de resultado e a melhora de 99 pacientes com TOC, tratados com 16 sessões semanais em quatro diferentes condições de tratamento: terapia cognitiva, EPR, fluvoxamina mais terapia cognitiva e fluvoxamina mais EPR. Não encontraram relação entre a situação profissional (se os pacientes estão empregados ou desempregados) e o resultado das diferentes condições de tratamento.

McLean et al. ${ }^{39}$ não observaram associação entre o nível educacional e a resposta aos tratamentos com TC ou TCC. Dos 63 pacientes que completaram os tratamentos, $5 \%$ tinham ensino médio incompleto, 52\% tinham formação universitária incompleta e 43\% completaram o programa equivalente a um curso universitário ou técnico.

Ferrão et al. ${ }^{43}$, em um estudo caso-controle, compararam 26 pacientes respondedores aos tratamentos com 23 refratários. Os respondedores deveriam ter redução ? 35\% no escore inicial da Yale-Brown Obsessive-Compulsive Scale (Y-BOCS) e ser classificados como "melhor" ou "muito melhor" na Clinical Global I mpression (CGI). Os refratários foram definidos por: redução < $25 \%$ no escore inicial da Y-BOCS após tratamento (ISRS por 16 semanas e 20 horas de TCC).

Os autores verificaram que um nível econômico mais baixo, pelos critérios da Associação Brasileira de Institutos de Pesquisa de Mercado (ABIPEME), foi associado à refratariedade aproximadamente $74 \%$ dos pacientes refratários pertenciam às classes C e D, contra $42 \%$ dos respondedores. A situação socioeconômica mais baixa poderia influenciar o nível educacional ou a situação profissional, formando uma interação entre características passíveis de exercer influência na resposta aos tratamentos, embora seja difícil a caracterização do que é causa ou efeito.

I dade do paciente

O único estudo encontrado no qual houve alguma associação entre a idade do paciente e o tratamento foi o ensaio clínico aberto de Foa et al. ${ }^{17}$, onde pacientes mais jovens apresentaram maior tendência de melhora após tratamento com TC e durante o seguimento. Os indivíduos considerados muito melhores tinham média de idade de 30,92 anos, contra 41,00 anos entre os que não mantiveram a melhora no seguimento. Entretanto, no modelo de regressão múltipla, esse fator não se relacionou significativamente com o resultado do tratamento.

Características intrínsecas do TOC

Gravidade inicial dos sintomas 
Para Basoglu et al. ${ }^{22}$, a gravidade da doença foi o preditor mais forte do resultado não-satisfatório no tratamento do TOC com TC e clomipramina. Os pacientes que inicialmente apresentaram um quadro mais grave da doença tiveram menor grau de habituação aos estímulos provocadores de rituais, e sua melhora foi menor. Pacientes moderadamente doentes melhoraram muito mais rapidamente no começo do tratamento, embora a presença de melhoras posteriores nos pacientes mais graves tenha atenuado esses efeitos.

Cottraux et al. ${ }^{25}$, em um estudo envolvendo TC e fluvoxamina, observaram que um aspecto da gravidade - a evitação, avaliada por um teste comportamental - foi forte preditor de resultados pobres com os tratamentos, já que isoladamente foi responsável por $68 \%$ dos insucessos, provavelmente em virtude de esses pacientes terem dificuldades em se submeter aos exercícios de EPR.

Keijsers et al. ${ }^{26}$, em um ensaio clínico randomizado, analisaram possíveis preditores de resultado em 40 pacientes tratados com TC. O tratamento teve duas fases, caracterizadas por oito sessões de TC e oito de prevenção de rituais, em um total de dois meses de duração. Verificaram que uma maior gravidade das queixas obsessivo-compulsivas era preditora de pior resposta.

Uma importante descoberta de De Haan et al. ${ }^{30}$ foi que $1 / 3$ dos pacientes considerados nãorespondedores após os tratamentos (TCC e/ou fluvoxamina) tornaram-se respondedores no seguimento. A característica principal destes pacientes foi a presença de queixas mais graves acerca dos SOC, confirmadas por um escore mais alto na Y-BOCS. A gravidade inicial dos sintomas predisse resultados mais pobres em curto prazo, embora muitos destes pacientes tenham melhorado no seguimento, indicando que estes precisariam de um período de terapia mais longo.

Steketee et al. ${ }^{35}$ avaliaram dados de seguimento de 5 anos de 100 pacientes com TOC, concluindo que uma gravidade menor inicial da doença foi preditora da remissão parcial dos sintomas. Aproximadamente $20 \%$ dos pacientes tiveram remissão total, e $50 \%$ tiveram remissão parcial durante o seguimento.

Hollander et al. ${ }^{41}$, na avaliação de 274 pacientes de oito centros internacionais, concluíram que a gravidade dos sintomas antes do início do tratamento era significativamente maior $(p=0,001)$ entre os pacientes refratários. Por outro lado, Foa et al. ${ }^{17}$ não encontraram qualquer relação entre gravidade inicial dos SOC e resultado da TC, seja no pós-tratamento, seja no seguimento.

Para Araújo et al. ${ }^{29}$, a gravidade inicial também não foi preditor de resultado à TC, muito embora uma gravidade inicial mais baixa tenha sido preditora de maior submissão às tarefas de casa de exposição na primeira semana de tratamento, a qual foi isoladamente o melhor preditor de bom resultado após TC e no seguimento de 32 semanas.

Em resumo, diversos estudos concluíram que uma maior gravidade inicial dos sintomas prediz piores resultados após os tratamentos do TOC $22,25,26,30,35,41$. Entretanto, algumas pesquisas indicaram que pacientes com sintomas mais graves obtêm benefícios com as terapias, embora respondam mais tardiamente e tendam a alcançar mais freqüentemente uma remissão parcial dos $\operatorname{sOC}^{22,30}$.

I dade de início dos sintomas

De Haan et al. ${ }^{30}$ não encontraram associação significativa entre a idade de início (antes ou após os 18 anos) dos sintomas e o resultado dos tratamentos do TOC com TCC, associada ou não à 


\section{fluvoxamina.}

Eisen et al. ${ }^{6}$, em um estudo prospectivo de 2 anos, analisaram possíveis padrões de remissão e de recaída no referido período, em 66 pacientes com TOC, também não encontrando efeito significativo da idade de início dos SOC no curso da doença. Os pacientes foram tratados com TC e inibidores de recaptação da serotonina (IRS).

Já Foa et al. ${ }^{17}$ encontraram associação entre essas variáveis. Assim, os pacientes cujos sintomas apareceram mais precocemente mantiveram seus ganhos com a TC de forma mais eficiente no seguimento do que os pacientes cujos sintomas apareceram mais tardiamente. A idade média de início dos SOC era de 23 anos.

Por outro lado, Skoog \& Skoog ${ }^{34}$, em um estudo de 40 anos de seguimento, analisaram os dados de 144 pacientes com TOC. Os autores observaram que o início precoce dos SOC foi preditor de piores resultados aos tratamentos.

Grant et al. ${ }^{44}$, em um estudo de coorte com 293 indivíduos com TOC, investigaram as características clínicas e comorbidades psiquiátricas dos 33 (11,3\%) pacientes com idade de início dos SOC acima de 29 anos. Concluíram que, embora o início do TOC com essa idade não seja comum, esses indivíduos possuem características clínicas similares às dos indivíduos com início precoce dos SOC e respondem melhor ao tratamento com TCC, provavelmente por apresentarem sintomas menos graves da doença ou até mesmo pela menor duração dos SOC nesse grupo antes de procurarem tratamento.

Em resumo, os achados são contraditórios, e a explicação para isso pode estar no fato de que a idade de início dos sintomas seja influenciada por outros fatores. O início precoce dos sintomas foi associado ao sexo masculino, à presença de maior número de obsessões e compulsões, à maior freqüência de rituais de repetição e à maior gravidade dos sintomas ${ }^{45}$; o grupo com início precoce dos sintomas apresentou maior associação com tiques, mais compulsões semelhantes a tiques e mais fenômenos sensoriais ${ }^{46}$. O grupo de início dos sintomas após os 29 anos (início tardio) apresentou menos obsessões de contaminação, somáticas e religiosas ${ }^{44}$. Existem controvérsias na definição do que seria considerado como idade de início precoce da doença, já que alguns autores consideram como sendo o TOC iniciado antes dos $10 \operatorname{anos}^{46}$, enquanto outros, antes dos 18 $\operatorname{anos}^{30,44}$.

\section{Duração da doença e tempo sem tratamento}

No único ensaio clínico encontrado, Keijsers et al. ${ }^{26}$ concluíram que uma maior duração da doença foi preditora de pior resultado para as obsessões. Contudo, esse achado não significa, necessariamente, que essa variável possa afetar o resultado da TC.

Ferrão et al. ${ }^{43}$ não encontraram diferenças significativas para a duração da doença: 22,27 anos para os pacientes refratários e 25,46 anos para os respondedores. Também não encontraram diferenças significativas entre esses dois grupos com relação ao período de tempo entre a idade de início dos sintomas e a idade de início do tratamento (tempo sem tratamento): 10,32 anos e 15,17 anos, respectivamente.

Em resumo, a duração da doença parece não ter um papel importante em determinar os resultados da TC e da TCC. Nesse sentido, estudos mais adequados devem ser conduzidos para apontar se o período de tempo sem nenhum tipo de tratamento poderia ser um fator preditivo de resposta. 


\section{Curso e história natural do TOC}

Em um estudo de seguimento de 40 anos, Skoog \& Skoog ${ }^{34}$ observaram que o curso crônico da doença, nos pacientes examinados entre 1954 e 1956, foi associado com pior prognóstico.

No estudo de Hollander et al. ${ }^{41}$, entre os pacientes que responderam aos tratamentos (TCC, TC ou IRS), 70\% apresentaram curso crônico da doença, contra mais de $90 \%$ entre os que não responderam. Os refratários apresentaram menos o início abrupto do TOC em comparação com os respondedores.

Entretanto, não fica claro se o curso crônico (e o desgaste causado por ele) acaba tornando o TOC refratário ou se o TOC refratário desde o início proporciona uma evolução mais crônica por características neurobiológicas próprias.

\section{Comorbidades}

As comorbidades psiquiátricas são bastante freqüentes no TOC, tendo sido encontrados 4, 6 diagnósticos ao longo da vida para pacientes com TOC, enquanto o grupo-controle recebeu, em média, apenas 1,2 diagnósticos ${ }^{47}$.

Minichiello et al. ${ }^{21}$ estudaram retrospectivamente dados de 29 pacientes tratados com TC e IRS. Na amostra, 10 pacientes tinham comorbidade com transtorno de personalidade esquizotípica. Daqueles pacientes sem tal comorbidade, 84\% melhoraram ao menos moderadamente com o tratamento (TC ou TC mais IRS), contra apenas uma melhora (10\%) entre os diagnosticados com transtorno de personalidade esquizotípica. A impressão clínica dos pesquisadores foi de que pacientes esquizotípicos têm dificuldades em focar uma tarefa específica, vindo a falhar na TC.

Cottraux et al. ${ }^{25}$ constataram que pacientes diagnosticados como tendo personalidade borderline ou paranóide não obtiveram bons resultados nos tratamentos do TOC com TC e/ou fluvoxamina.

No estudo de seguimento de 5 anos de Steketee et al. ${ }^{35}$, dos 100 pacientes iniciais, $76 \%$ tinham ao menos uma comorbidade: 36\% com episódio depressivo maior (EDM), 32\% com transtorno de ansiedade generalizada, $27 \%$ com fobia social e 37\% com pânico e agorafobia. Somente o EDM tendeu $(p=0,10)$ a ser um preditor de pior curso da doença. Os transtornos de ansiedade não influenciaram o curso do TOC. Quanto aos transtornos de personalidade, nem o número nem a presença de um transtorno de personalidade foram preditores do curso do TOC.

Marks et al. ${ }^{19}$, em um ensaio clínico randomizado, estudaram 40 pacientes com TOC que foram tratados em dois diferentes grupos: um recebeu clomipramina, enquanto o outro recebeu placebo. Durante 4 semanas, um grupo recebeu sessões de relaxamento, e o outro foi tratado com técnicas de EPR. Após, durante mais 4 semanas, ambos foram tratados com EPR. Os autores perceberam que a TC produziu menores mudanças no humor, concluindo que a EPR é uma das escolhas de tratamento do TOC para pacientes não-deprimidos.

Keijsers et al. ${ }^{26}$ detectaram que maiores níveis de depressão - avaliada pela Hamilton Depression Rating Scale (HAM-D) - foram preditores de resultados mais pobres para as compulsões, afetando negativamente o tratamento com a TC. 
Abramowitz et al. ${ }^{37}$ examinaram os efeitos da depressão na resposta ao tratamento do TOC (TCC ou TCC mais anti-obsessivos). Os 87 pacientes foram divididos em grupos, conforme os escores que apresentavam no pré-tratamento, obtidos pelo Beck Depression Inventory (BDI). Aqueles com depressão inicial grave (BDI ? 30) melhoraram significativamente menos comparados com os menos deprimidos ou com os não-deprimidos, mesmo tendo obtido ganhos com o tratamento. Os pacientes com depressão grave falham nos exercícios de exposição e têm menos motivação para tratamento, sendo prováveis causas do pior resultado obtido, conforme os pesquisadores.

Já Foa et al. ${ }^{24}$ testaram a hipótese de que a resposta à TC poderia ser melhorada com a redução do grau de depressão antes do início da TC. Por meio de um ensaio clínico randomizado, 38 pacientes com TOC foram divididos em dois grupos, cujo escore de corte na BDI foi de 20 . Metade de cada grupo recebeu imipramina, e a outra metade recebeu placebo durante 6 semanas. Após, todos realizaram TC diariamente durante 3 semanas, seguida de 12 sessões semanais de psicoterapia de reforço. Embora a imipramina tenha melhorado os sintomas depressivos, nem o nível de depressão nem o efeito antidepressivo do medicamento influenciaram o resultado após os tratamentos e no seguimento de 2 anos, indicando que a depressão seria decorrente dos SOC.

De Haan et al. ${ }^{30}$ não consideraram a depressão como variável preditora de resultado das terapias (TCC e/ou fluvoxamina). Também não foi associada ao resultado após tratamento de 34 pacientes com TC e clomipramina, nem no seguimento de 6 anos no estudo de O'Sullivan et al. ${ }^{23}$.

Com relação ao transtorno de tiques (TT) e à síndrome de Tourette (ST), Miguel et al. ${ }^{48,49}$ sugeriram um continuum entre os transtornos dos movimentos repetitivos, variando dos tiques simples à ideação obsessiva, estando inseridos nesse modelo os chamados transtornos do espectro do TOC, como o TT ou a ST. Estes ocorrem mais no grupo de pacientes que têm TOC de início precoce, na maioria em homens, com maior associação com histórico familiar positivo para tiques e com padrão distinto de resposta aos tratamentos ${ }^{50}$, já que tendem a responder melhor com medicação neuroléptica associada aos ISRS do que com TC ou TCC.

Hollander et al. ${ }^{41}$ evidenciaram que aqueles pacientes que não responderam aos tratamentos ( $\mathrm{n}=$ 147) estavam entre os que possuíam mais comorbidades com transtornos alimentares e bipolar; porém, nesse último caso, a análise estatística demonstrou apenas tendência $(p<0,09)$. Também encontraram um resultado não esperado, pois os pacientes respondedores $(n=127)$ tiveram maiores taxas de histórico familiar com tiques $(p=0,024): 7,5 \%$ de freqüência contra apenas $1,6 \%$ entre os não-respondedores.

Em resumo, existem evidências de estarem associados com mau resultado às psicoterapias principalmente o transtorno de personalidade esquizotípica, além do paranóide e borderline. A depressão grave associada ao TOC parece afetar negativamente o resultado dos tratamentos. Entretanto, não há unanimidade nesse achado, já que alguns estudos não encontraram relação ${ }^{23,30}$. Os indivíduos com ST e TT tendem a responder melhor com medicação neuroléptica associada aos ISRS. É possível que todas essas comorbidades interfiram na motivação e na adesão ao tratamento e até mesmo na capacidade do paciente de se vincular ao terapeuta.

Tipos de sintomas

Black et al. ${ }^{32}$, em um ensaio clínico randomizado com 38 pacientes de TOC, analisaram fatores associados à resposta aos tratamentos com paroxetina ou TCC. A resposta foi avaliada por redução na Y-BOCS ? 40\% e classificação na CGI como "melhores" ou "muito melhores". O colecionismo constituiu-se em um importante sintoma preditor de resultados pobres em pacientes com TOC tratados com TCC. 
Saxena et al. ${ }^{40}$ estudaram retrospectivamente 190 pacientes com TOC tratados, entre 1994 e 2000, por 6 semanas com TCC intensiva, medicação e reabilitação psicossocial (informações sobre diagnóstico, medicação, prevenção de recaídas). Vinte pacientes (11\%) pertenciam ao agrupamento dos colecionistas (definido através da Y-BOCS Symptom Checklist). Eles eram significativamente mais velhos (média de idade de 44,8 versus 33,2 anos), com maior proporção de mulheres (15 de 20 pacientes), e apresentavam maiores níveis de ansiedade antes e após os tratamentos. Os pacientes "não-colecionistas" melhoraram significativamente mais (Y-BOCS médio final de 15,6 contra 19,9 ) que os colecionadores.

Rufer et al. ${ }^{42}$ examinaram quais dimensões dos sintomas do TOC poderiam predizer resultados no tratamento com quatro sessões semanais de TCC, durante 9 semanas, de 104 pacientes. Os sintomas foram avaliados através da Y-BOCS Symptom Checklist, sendo que somente sete dos 19 pacientes $(36,8 \%)$ com sintomas de colecionismo responderam à TCC, enquanto 47 dos 75 pacientes não-colecionistas $(62,7 \%)$ responderam. Os pacientes com obsessões de conteúdo sexual/religioso também tenderam a responder menos, embora o resultado não tenha sido significativo $(p=0,07)$.

Alonso et al. ${ }^{38}$ analisaram dados de 60 pacientes com TOC. Todos receberam inicialmente tratamento medicamentoso (IRS) por 12 semanas e, após, 20 sessões semanais de uma hora de TC. Cerca de $17 \%$ dos pacientes tiveram ao menos um sintoma na dimensão de conteúdo sexual/religioso, considerada como sendo a principal. Eles tiveram pior resultado no seguimento de até 5 anos, constituindo-se essa dimensão um preditor de mau prognóstico de longo prazo.

Ferrão et al. ${ }^{43}$ utilizaram o instrumento Dimensional Y-BOCS (DYBOCS) para medir a gravidade em cada uma das seis dimensões de sintomas. Encontraram que a dimensão de conteúdo sexual/religioso foi associada à refratariedade. Cerca de $73 \%$ dos pacientes refratários estavam incluídos nessa dimensão, contra $38 \%$ dos respondedores.

Em resumo, obsessões e compulsões relacionadas ao colecionismo ou de conteúdo sexual/religioso foram as que mais apareceram relacionadas a uma pior resposta aos tratamentos do TOC. $\mathrm{O}$ dimensionamento dos sintomas pode ser útil para identificar subgrupos de pacientes, confirmando a natureza multidimensional e heterogênea da patologia.

Fatores cognitivos

Juízo crítico (insight) e idéias supervalorizadas

Neziroglu et al. ${ }^{33}$, num estudo relacionando a presença de idéias supervalorizadas com a resposta à TC ou TCC, avaliaram 20 pacientes, sendo 15 homens portadores de TOC. Na admissão, os pacientes foram divididos em dois grupos: um formado por 10 indivíduos com presença de alto grau de idéias supervalorizadas - Overvalued Ideas Scale (OVIS) ? 6 - e outro com baixo grau. Todos ingressaram em um programa de tratamento hospitalar intensivo de EPR com duração de 1 mês, usavam ISRS com doses estabilizadas (? 3 meses) e continuaram a usá-los durante o tratamento. Os resultados indicaram que pacientes com altos escores na OVIS sofrem interferências no tratamento desde o início, não ocorrendo mudanças nos níveis de ansiedade e depressão com o tratamento. Os autores concluíram que a presença de idéias supervalorizadas constitui-se em mau prognóstico do TOC.

Foa et al. ${ }^{36}$ examinaram o grau em que a presença do insight pobre acerca dos SOC influenciou a eficácia da TC no tratamento do TOC. Vinte pacientes receberam tratamento com técnicas de EPR, sendo que aqueles que tinham presença de idéias supervalorizadas, com a certeza de que seus 
medos não eram desprovidos de sentido, obtiveram piores resultados com a terapia do que os outros pacientes, apesar da redução dos níveis de fixidez das suas crenças.

No estudo de Hollander et al. ${ }^{41}$, entre os pacientes que responderam aos tratamentos (TCC, TC ou IRS), $10 \%$ tinham juízo crítico pobre, contra $20 \%$ entre os que não responderam $(p<0,025)$.

O insight pobre constitui um subtipo do $\mathrm{TOC}^{1}$. Os pacientes não consideram seus sintomas sem sentido ou excessivos. Poderia haver uma acomodação ao sintoma, levando esses pacientes a procurarem atendimento mais tardiamente, criando uma relação entre juízo crítico pobre e maior duração da doença ou maior tempo sem tratamento.

Adesão ao tratamento, motivação e funcionamento familiar

Keijsers et al. ${ }^{26}$ verificaram que uma menor motivação para o tratamento e uma insatisfação com a relação terapêutica foram preditores de resultados mais pobres para as obsessões, afetando o resultado no tratamento do TOC com TC. A motivação foi avaliada através da subescala "disposição em participar" da Nijmegen Motivation List (NML). A qualidade da relação terapêutica foi avaliada através de quatro subescalas do Relationship Inventory (RI).

Araújo et al. ${ }^{29}$ constataram que o melhor preditor de bom resultado no final do tratamento do TOC com TC e no seguimento de 32 semanas foi a adesão inicial às tarefas de casa de exposição durante a primeira semana de tratamento. A adesão foi definida como o percentual completado comparado com o número de tarefas de casa combinado entre paciente e terapeuta. A implicação clínica principal do estudo é que pacientes com TOC que realizam de forma apropriada as tarefas de auto-exposição e de prevenção de rituais, na primeira semana de tratamento, são os que mais provavelmente serão bem-sucedidos 7 meses mais tarde.

De Haan et al. ${ }^{30}$ demonstraram que a motivação foi preditor de um resultado mais favorável dos tratamentos (TCC e/ou fluvoxamina). Foi utilizado o instrumento NML para aferir motivação.

Ferrão et al. ${ }^{43}$ avaliaram o comportamento de familiares de pacientes com TOC, nos quais aplicaram o instrumento Family Accommodation Scale (FAS), constatando que o grupo de familiares dos pacientes refratários apresentava maior índice de acomodação e maior índice de desgaste familiar quando comparado aos familiares de pacientes respondedores. Quanto maior for a modificação do funcionamento familiar devido ao TOC - se os familiares compartilham dos rituais ou mesmo se forem hostis aos mesmos -, maiores serão os escores na FAS, característica associada à refratariedade.

Em resumo, adesão e motivação são fatores que podem interferir no desempenho dos tratamentos, já que constituem características importantes para o bom desempenho nas técnicas de EPR. A aliança que os familiares acabam formando com o paciente nos seus rituais também interfere nos tratamentos, uma vez que pode ajudar a perpetuar os sintomas.

Aspectos do tratamento

Tratamentos anteriores 
Foi encontrado apenas um estudo que associou diretamente a ausência ou a presença de tratamento anterior para o TOC com o desempenho alcançado na realização de uma nova terapia. Buchanan et al. ${ }^{28}$ estudaram fatores associados com a melhora clínica e a adesão ao tratamento do TOC com técnicas de EPR. Todos os 127 pacientes do estudo foram acompanhados pelo período de 12 meses. Aqueles que não haviam se tratado anteriormente tiveram melhoras clínicas mais robustas.

De Haan et al. ${ }^{30}$ observaram que os respondedores tardios (pacientes que não responderam após a terapia, mas que se tornaram respondedores no seguimento) haviam recebido mais freqüentemente tratamentos anteriores para o TOC $(p<0,05)$. No entanto, essa variável não foi analisada como possível preditora de resultado dos tratamentos.

A realização de tratamento anterior poderia sugerir um paciente com melhor juízo crítico ou maior gravidade dos SOC, motivando a procura anterior por ajuda, indicando, inclusive, um paciente refratário ao tratamento já realizado.

Grau de melhora, remissão completa e melhora precoce

Mawson et al. ${ }^{20}$, em um ensaio clínico randomizado, estudaram 40 pacientes com TOC que receberam tratamento com técnicas de EPR e clomipramina. Verificaram que as maiores associações com melhor resultado no seguimento de 2 anos foram um tempo mais prolongado de EPR e uma resposta ao tratamento nas $10^{\underline{a}}$ e $18^{\underline{a}}$ semanas.

No estudo de FOA et al. ${ }^{17}$, os pacientes com ganhos intermediários logo após o tratamento com TC apresentaram alto risco de recaída durante o seguimento. I sso sugere que a satisfação com melhora parcial é injustificada, devendo-se realizar esforços no intuito de atingir a redução completa dos SOC no final do tratamento.

O'Sullivan et al. ${ }^{23}$, num estudo de seguimento de 6 anos, analisaram dados de 34 pacientes que participaram de um ensaio clínico randomizado, envolvendo a técnica de exposição por 3 ou 6 semanas, além da utilização de clomipramina ou placebo por 36 semanas. Os autores constataram que o grau de melhora após TC, além de uma maior exposição ao tratamento, foram preditores de melhor prognóstico.

Braga et al. ${ }^{13}$ avaliaram os resultados da TCC em grupo e a existência de fatores preditores de resultado a longo prazo em 42 pacientes com TOC. A intensidade da melhora dos SOC e sua remissão completa, definida por escores nas escalas Y-BOCS ? 8 e CGI <2, foram fortes fatores preditores para a não-recaída no seguimento de 1 ano. Nenhum paciente com remissão total recaiu no seguimento.

Em resumo, o grau de melhora com os tratamentos e a remissão completa dos sintomas parecem exercer papel fundamental na não-recaída dos pacientes.

\section{Fatores neurobiológicos}

Baxter et al. ${ }^{51}$ utilizaram tomografia cerebral por emissão de pósitrons para investigar taxas do metabolismo da glicose em pacientes com TOC, antes e após tratamento com TC ou fluoxetina. Concluíram que a hiperatividade diminui no núcleo caudado, nos lobos órbito-frontais e no córtex 
As características intrínsecas do TOC são complexas, podendo a patologia ser entendida como um grupo heterogêneo de transtornos. Existem evidências de que o início precoce dos SOC indique pior prognóstico, apesar de não haver unanimidade e de um estudo ter encontrado resultado distinto pacientes cujos sintomas apareceram mais precocemente mantiveram seus ganhos com a TC de modo mais eficiente ${ }^{17}$. Os diferentes resultados poderiam ser explicados pelas distintas formas da definição de início precoce e pelo fato de tal variável ser influenciada por outros fatores. 0 comportamento evitativo grave, embora pouco estudado, pode ser responsável pelo insucesso dos tratamentos, especialmente com a técnica de $\mathrm{EPR}^{25}$. A gravidade maior dos sintomas estaria relacionada com resultados mais pobres em curto prazo, embora não signifique que esses pacientes sejam refratários aos tratamentos, necessitando, talvez, de abordagens mais longas. Existem evidências de estarem associados com mau resultado principalmente o transtorno de personalidade esquizotípico, além do paranóide e do borderline. É possível que essas condições interfiram na motivação e na adesão ao tratamento, bem como na capacidade do paciente de se vincular ao terapeuta. A comorbidade com TT e ST está associada com piores resultados aos tratamentos (psicoterapias ou ISRS). Não encontramos estudos que avaliassem possível relação do abuso de substâncias, inclusive álcool, provavelmente pelo fato de essa comorbidade ser, com freqüência, critério de exclusão nos tratamentos. Quanto à depressão, a evidência que dispomos, e se restringe a um único artigo, é que tratá-la antes do TOC não influenciou a resposta à TC ${ }^{24}$. O curso crônico da doença foi relacionado a piores prognósticos, embora não fique claro se o desgaste causado por este acabe tornando o TOC refratário ou se o TOC refratário proporcione uma evolução mais crônica, devido a características neurobiológicas próprias. A avaliação dimensional dos SOC pode ser útil para identificar subgrupos de pacientes, confirmando a natureza multidimensional e heterogênea da patologia. O colecionismo e os sintomas de conteúdo sexual/religioso estão relacionados a uma pior resposta aos tratamentos do TOC.

O juízo crítico pobre poderia tornar os pacientes egossintônicos, levando-os a procurar atendimento mais tarde, já que ocorre uma acomodação ao sintoma. A expectativa, a motivação e a adesão ao tratamento seriam características favoráveis, assim como a maneira como os que familiares do paciente comportam-se diante dos sintomas da doença, podendo ajudar a perpetuálos, caso entrem em conluio com os rituais. Não há unanimidade se os fatores cognitivos interferem na resposta aos tratamentos, em decorrência, provavelmente, das diferentes formas de aferição utilizadas nas pesquisas.

Os aspectos do tratamento exercem papel fundamental na predição de resultado. A intensidade da melhora dos $\mathrm{SOC}^{13}$ e sua remissão completa ${ }^{13,17}$ foram fortes preditores para a não-recaída no seguimento de até 2 anos. Inclusive nenhum paciente com remissão total no final do tratamento recaiu no seguimento ${ }^{13}$. Uma das principais conclusões da 6a Conferência Internacional do TOC foi que a satisfação com a melhora parcial é injustificada, devendo-se realizar esforços no intuito de se atingir redução total dos SOC no final do tratamento. Não encontramos estudos que avaliem as qualidades do terapeuta, como empatia, segurança e respeito, as quais poderiam interferir na dinâmica e no resultado dos tratamentos.

Os fatores neurobiológicos estão sendo cada vez mais estudados com o desenvolvimento dos equipamentos que rastreiam o funcionamento cerebral, sendo fundamentais para a compreensão da fisiopatogenia do TOC. Já foram encontradas diferenças anatômicas e metabólicas, sugerindo que indivíduos com diferentes padrões podem responder preferencialmente a um tipo ou outro de tratamento 31,51 .

Em que pese o grande número de pesquisas que tentam identificar fatores associados à melhora ou não dos sintomas com as terapias de EPR e TCC, essa questão está longe de ser esclarecida. As razões para tais dificuldades podem estar relacionadas à heterogeneidade do TOC e das amostras utilizadas nos diferentes estudos, além da não-padronização das técnicas psicoterápicas utilizadas. Outra explicação seria que, nas situações clínicas, múltiplos fatores agem em conjunto para produzir efeitos. As variáveis podem relacionar-se entre si, além de se relacionarem com o 
desfecho de interesse. O efeito de uma pode ser modificado pela presença de outras, e a soma dos efeitos de duas ou mais variáveis pode ser maior que a soma de seus efeitos individuais ${ }^{52}$. Por fim, é possível que fatores não-específicos relacionados com a pessoa do terapeuta, a qualidade da relação terapêutica, além da motivação e da capacidade de tolerar frustração por parte do paciente, possam exercer um papel importante que não tem sido avaliado pelas pesquisas.

\section{REFERÊNCIAS}

1. American Psychiatric Association. Manual diagnóstico e estatístico de transtornos mentais: DSMIV-TR. 4a ed rev. Porto Alegre: Artmed; 2002.

2. Kessler RC, Berglund P, Demler O, J in R, Merikangas KR, Walters EE. Lifetime prevalence and age-of-onset distributions of DSM-IV disorders in the national comorbidity survey replication. Arch Gen Psychiatry. 2005; 62(6): 593-602.

3. Lensi P, Cassano GB, Correddu G, Ravagli S, Kunovac JL, Akiskal HS. Obsessive-compulsive disorder. Familial-developmental history, symptomatology, comorbidity and course with special reference to gender-related differences. Br J Psychiatry. 1996; 169(1): 107-7.

4. March JS, Frances A, Kahn DA, Carpenter D. The expert consensus guideline series: treatment of obsessive-compulsive disorder. J Clin Psychiatry. 1997; 58(Suppl 4): 3-72.

5. Clomipramine in the treatment of obsessive-compulsive disorder. The Clomipramine Collaborative Study Group. Arch Gen Psychiatry. 1991;48(8): 730-8.

6. Eisen J L, Goodman WK, Keller MB, Warshaw MG, De Marco LM, Luce DD, et al. Patterns of remission and relapse in obsessive-compulsive disorder: a 2-year prospective study. J Clin Psychiatry. 1999;60(5):346-51.

7. Pigott T, Seay S. Pharmacotherapy of obsessive-compulsive disorder: overview and treatmentrefractory strategies. In: Goodman WK, Rudorfer MV, Maser JD. Obsessive-compulsive disorder contemporary issues in treatment. London: Lawrence Erlbaum Associates; 2000. p. 277-82.

8. Pato MT, Zohar-Kadouch R, Zohar J, Murphy DL. Return of symptoms after discontinuation of clomipramine in patients with obsessive-compulsive disorder. Am J Psychiatry. 1988; 145

(12): 1521-5.

9. Foa EB. Failures in treating obsessive-compulsives. Behav Res Ther. 1979; 17: 169: 76.

10. Abramowitz J. Effectiveness of psychological and pharmacological treatments for obsessivecompulsive disorder: a quantitative review. J Consult Clin Psychol. 1997;65(1):44-52.

11. Asbahr FR, Castillo AR, I to LM, Latorre MR, Moreira MN, Lotufo-Neto F. Group cognitivebehavioral therapy versus sertraline for the treatment of children and adolescents with obsessivecompulsive disorder. J Am Acad Child Adolesc Psychiatry. 2005;44(11): 1128-36.

12. Souza MB, Isolan LR, Oliveira RR, Manfro GG, Cordioli AV. A randomized clinical trial of cognitive-behavioral group therapy and sertraline in the treatment of obsessive-compulsive disorder. J Clin Psychiatry. 2006;67(7): 1133-9.

13. Braga DT, Cordioli AV, Niederauer K, Manfro GG. Cognitive-behavioral group therapy for 
obsessive-compulsive disorder: a 1-year follow-up. Acta Psychiatr Scand. 2005:112(3): 180-6.

14. Falls-Stewart W, Marks AP, Schafer J. A comparison of behavioral group therapy and individual behavior therapy in treating obsessive-compulsive disorder. J Nerv Ment Dis. 1993; 181(3): 189-93.

15. Emmelkamp PMG, Bouman TK, Blaauw E. Individualized versus standardized therapy: a comparative evaluation with obsessive-compulsive patients. Clin Psychol Psychother. 1994; 1: 95 100.

16. Volpato Cordioli A, Heldt E, Braga Bochi D, Margis R, Basso de Sousa M, Fonseca Tonello J, et al. Cognitive-behavioral group therapy in obsessive-compulsive disorder: a randomized clinical trial. Psychother Psychosom. 2003; 72(4):211-6.

17. Foa EB, Grayson JB, Steketee GS, Doppelt HG, Turner RM, Latimer PR. Success and failure in the behavioral treatment of obsessive-compulsives. J Consult Clin Psychol. 1983;51(2):287-97.

18. Cordioli AV, Heldt E, Bochi DB, Margis R, Sousa MB, Tonello JF, et al. Cognitive-behavioral group therapy in obsessive-compulsive disorder: a clinical trial. Rev Bras Psiquiatr. 2002;24 (3): 113-20.

19. Marks IM, Stern RS, Mawson D, Cobb J, McDonald R. Clomipramine and exposure for for obsessive-compulsive rituals. Br J Psychiatry. 1980; 136: 1-25.

20. Mawson D, Marks IM, Ramm L. Clomipramine and exposure for chronic obsessive-compulsive rituals: III. Two year follow-up and further findings. Br J Psychiatry. 1982; 140: 11-18.

21. Minichiello WE, Baer L, Jenike MA. Schizotypal personality disorder: a poor prognostic indicator for behavior therapy in the treatment of obsessive-compulsive disorder. J Anx Disorders.

$1987 ; 1: 273-6$.

22. Basoglu M, Lax T, Kasvikis Y, Marks IM. Predictors of improvement in obsessive-compulsive disorder. J Anx Disorders. 1988;2:299-317.

23. O'Sullivan G, Noshirvani H, Marks I, Monteiro W, Lelliott P. Six-year follow-up after exposure and clomipramine therapy for obsessive-compulsive disorder. J Clin Psychiatry. 1991;52(4):150-5.

24. Foa EB, Kozak MJ , Steketee GS, McCarthy PR. Treatment of depressive and obsessivecompulsive symptoms in OCD by imipramine and behaviour therapy. Br J Clin Psychol. 1992;31(Pt 3): 279-92.

25. Cottraux J, Messy P, Marks IM, Mollard E, Bouvard M. Predictive factors in the treatment of obsessive-compulsive disorders with fluvoxamine and or behavior therapy. Behav Psychother. 1993; $21: 45-50$.

26. Keijsers GP, Hoogduin CA, Schaap CP. Predictors of treatment outcome in the behavioural treatment of obsessive-compulsive disorder. Br J Psychiatry. 1994;165(6): 781-6.

27. Castle DJ, Deale A, Marks IM, Cutts F, Chadhoury Y, Stewart A. Obsessive-compulsive disorder: prediction of out-come from behavioural psychotherapy. Acta Psychiatr Scand. 1994; 89(6):393-8.

28. Buchanan AW, Meng KS, Marks IM. What predicts improvement and compliance during the behavioral treatment of obsessive compulsive disorder? Anxiety. 1996;2(1):22-7.

29. de Araújo LA, I to LM, Marks IM. Early compliance and other factors predicting outcome of exposure for obsessive-compulsive disorder. Br J Psychiatry. 1996; 169(6): 747-52. 
30. de Haan E, van Oppen P, van Balkom AJ, Spinhoven P, Hoogduin KA, Van Dyck R. Prediction of outcome and early vs. late improvement in OCD patients treated with cognitive behaviour therapy and pharmacotherapy. Acta Psychiatr Scand. 1997; 96(5):354-61.

31. Brody AL, Saxena S, Schwartz JM, Stoessel PW, Maidment K, Phelps ME, et al. FDG-PET predictors of response to behavioral therapy versus pharmacotherapy in obsessive-compulsive disorder. Psychiatry Res Neuroimaging. 1998;84(1): 1-6.

32. Black DW, Monahan P, Gable J, Blum N, Clancy G, Baker P. Hoarding and treatment response in 38 nondepressed subjects with obsessive-compulsive disorder. J Clin Psychiatry. 1998;59

(8): 420-5.

33. Neziroglu FA, Stevens KP, Yaryura-Tobias JA. Overvalued ideas and their impact on treatment outcome. Rev Bras Psiquiatr. 1999;21(4):209-16.

34. Skoog G, Skoog I. A 40-year follow-up of patients with obsessive-compulsive disorder. Arch Gen Psychiatry. 1999; 56: 121-7.

35. Steketee G, Eisen J, Dyck I, Warshaw M, Rasmussen S. Predictors of course in obsessivecompulsive disorder. Psychiatry Res. 1999; 89(3):229-38.

36. Foa EB, Abramowitz JS, Franklin ME, Kosak MJ. Feared consequences, fixity of belief, and treatment outcome in patients with obsessive-compulsive disorder. Behav Ther. 1999; 30(4): 71724.

37. Abramowitz JS, Franklin ME, Street GP, Kosak MJ, Foa EB. Effects of comorbid depression on response to treatment for obsessive-compulsive disorder. Behav Ther. 2000;31(3):517-28.

38. Alonso P, Menchon J M, Pifarre J, Mataix-Cols D, Torres L, Salgado P, et al. Long-term follow-up and predictors of clinical outcome in obsessive-compulsive patients treated with serotonin reuptake inhibitors and behavioral therapy. J Clin Psychiatry. 2001;62(7):535-40.

39. McLean PD, Whittal ML, Thordarson DS, Taylor S, Söchting I, Koch WJ, et al. Cognitive versus behavior therapy in the group treatment of obsessive-compulsive disorder. J Consult Clin Psychol. 2001; 69: 205-14.

40. Saxena S, Maidment KM, Vapnik T, Golden G, Rishwain T, Rosen RM, et al. Obsessivecompulsive hoarding: symptom severity and response to multimodal treatment. J Clin Psychiatry. 2002;63(1):21-7.

41. Hollander E, Bienstock CA, Koran LM, Pallanti S, Marazziti D, Rasmussen SA, et al. Refractory obsessive-compulsive disorder: state-of-the-art treatment. J Clin Psychiatry. 2002;63 Suppl 6:209.

42. Rufer M, Fricke S, Moritz S, Kloss M, Hand I. Symptom dimensions in obsessive-compulsive disorder: prediction of cognitive-behavior therapy outcome. Acta Psychiatr Scand. 2006;113 (5): 440-6.

43. Ferrão YA, Shavitt RG, Bedin NR, de Mathis ME, Carlos Lopes A, Fontenelle LF, et al. Clinical features of refractory obsessive-compulsive patients. J Affect Disord. 2006;94(1-3): 199-209.

44. Grant JE, Mancebo MC, Pinto A, Williams KA, Eisen J L, Rasmussen SA. Late-onset obsessive compulsive disorder: clinical characteristics and psychiatric comorbidity. Psychiatry Res. 2007; 152 (1):21-7.

45. Fontenelle LF, Marques C, Versiani M. The effect of gender on the clinical features and 
therapeutic response in obsessive-compulsive disorder. Rev Bras Psiquiatr. 2002;24(1): 7-11.

46. Rosário-Campos MC, Leckman JF, Mercadante MT, Shavitt RG, Prado HS, Sada P, et al. Adults with early-onset obsessive-compulsive disorder. Am J Psychiatry. 2001; 158(11): 1899-903.

47. Miranda MA. Transtorno obsessivo-compulsivo e comorbidade: um estudo caso-controle [tese]. São Paulo (SP): Universidade Federal de São Paulo, Escola Paulista de Medicina; 1999.

48. Miguel EC, Coffey BJ, Baer L, Savage CR, Rauch SL, Jenike MA. Phenomenology of intentional repetitive behaviors in obsessive-compulsive disorder and Tourette's syndrome. J Clin Psychiatry. $1995 ; 56(6): 256-55$.

49. Miguel EC, Baer L, Coffey BJ, Rauch SL, Savage CR, O'Sullivan RL, et al. Phenomenological differences appearing with repetitive behaviors in obsessive-compulsive disorder and Gilles de la Tourette syndrome. Br J Psychiatry. 1997; 170: 140-5.

50. Miguel EC, do Rosário-Campos MC, Shavitt RG, Hounie AG, Mercadante MT. The tic-related obsessive-compulsive disorder phenotype. Adv Neurol. 2001; 85:43-55.

51. Baxter LR, Schwartz J M, Bergman KS, Szuba MP, Guze BH, Mazziotta J C, et al. Caudate glucose metabolic rate changes with both drug and behavior therapy for obsessive-compulsive disorder. Arch Gen Psychiatry. 1992; 49(9):681-9.

52. Fletcher RH, Fletcher SW, Wagner EH. Epidemiologia clínica: elementos essenciais. 3rd ed. Porto Alegre: Artmed; 1996.

\section{囵 Correspondência}

Andréa Litvin Raffin

Hospital de Clínicas de Porto Alegre

Rua Ramiro Barcelos, 2350, sala 400N

CEP 90035-903, Porto Alegre, RS.

Tel: (51) 3316.8294

Fax: (51) 3316.8493

E-mail:alitvin@terra.com.br

Recebido em 02/03/2007.

Aceito em 29/05/2007.

* Apoio financeiro: A primeira autora conta com bolsa de mestrado fornecida pela Coordenação de Aperfeiçoamento de Pessoal de Nível Superior (CAPES) e apoio do Fundo de Incentivo à Pesquisa e Eventos (FIPE) do Hospital de Clínicas de Porto Alegre.

Não foram declarados conflitos de interesse associados à publicação deste artigo. 